\title{
Transmission Electron Microscope Specimen Preparation of Si-Based Anode Materials for Li-lon Battery by Using Focused Ion Beam and Ultramicrotome
}

\author{
Jeong Eun Chae, Jun Mo Yang ${ }^{1}$, Sung Soo Kim², Ju Cheol Park* \\ Testing \& Certification Division Materials Characterization Center, Gumi Electronics \& Information Technology Research Institute, \\ Gumi 39171, Korea \\ ${ }^{1}$ Department of Nanostructure Technology, National Nanofab Center, Daejeon 34141, Korea \\ ${ }^{2}$ Graduate School of Energy Science and Technology, Chungnam National University, Daejeon 34134, Korea
}

*Correspondence to:

Park JC,

(iD http://orcid.org/0000-0002-9489-1638

Tel: +82-54-479-2181

Fax: +82-54-479-2080

E-mail: jcpark13@geri.re.kr

Received April 11, 2018

Revised April 27, 2018

Accepted April 28, 2018
A successful transmission electron microscope (TEM) analysis is closely related to the preparation of the TEM specimen and should be followed by the suitable TEM specimen preparation depending on the purpose of analysis and the subject materials. In the case of the Si-based anode material, lithium atoms of formed Li silicide were removed due to ion beam and electron beam during TEM specimen preparation and TEM observation. To overcome the problem, we proposed a new technique to make a TEM specimen without the ion beam damage. In this study, two types of test specimens from the Si-based anode material of Li-ion battery were prepared by respectively adopting the only focused ion beam (FIB) method and the new FIB-ultramicrotome method. TEM analyses of two samples were conducted to compare the Ga ion damage of the test specimen.

Key Words: Li ion battery, Si-based anode material, Focused ion beam, Ultramicorome, Transmission electron microscopy

\section{INTRODUCTION}

As Li-ion batteries have been popular as the next-generation energy source, various promising electrode materials have been investigated to improve battery performance. Especially, silicon appears as one of the most prominent anode materials for Li-ion batteries due to higher theoretical capacity and lower working potential. However, silicon experiences a huge volume expansion during lithiation and delithiation process, which causes rapid capacity degradation and very poor cycling performance (Chan et al., 2008; Obrovac \& Christensen, 2004; Park et al., 2010; Winter et al., 1998). In order to solve these problems, material researches like nanostructures and amorphous $\mathrm{Si}$ and Si-alloy materials combined with matrix and have been investigated (Jung et al., 2011; Lee \& Lee, 2002; Mao et al., 1999). In order to understand the reaction mechanism of Li and silicon crystal structures, obvious and direct observation method is very important. In this paper, we investigated the change of microstructure of Si alloy anode materials composed of active $\mathrm{Si}$ and inactive $\mathrm{TiFeSi}_{2}$ matrix after Li charging by using transmission electron microscope (TEM).

A success in the TEM analysis is closely related to the quality of TEM specimen. Therefore, the TEM specimen should be differently prepared in accordance with the purpose of analysis and target materials. There are various TEM specimen preparation techniques such as conventional ionmilling method, ultramicrotome and focused ion beam (FIB). TEM specimen of electrode materials for Li-ion battery are generally prepared by the FIB which can make the specimen thickness uniform and make it possible to easily observe a cross-section view of specific area of interest. In the case of the cathode material, Li bonding is strong, so there is no problem in TEM specimen preparation and

(a) This is an open-access article distributed under the terms of the Creative Commons Attribution Non-Commercial License (http://creativecommons.org/licenses/by-nc/4.0) which permits unrestricted noncommercial use, distribution, and reproduction in any medium, provided the original work is properly cited.

Copyrights @ 2018 by Korean Society of Microscopy 
TEM observation. However, in the case of the Si-based anode material, lithium atoms of formed $\mathrm{Li}$ silicide were removed due to ion beam and electron beam during TEM specimen preparation and TEM observation (Danet et al., 2010). To overcome the problem, TEM specimens need to be prepared using mechanical techniques without ion beam damage. Although the specimen made by FIB has damages induced by $\mathrm{Ga}$ ions, the one made by ultramicrotome has artifacts such as plastic deformation and tears of TEM specimen due to mechanical force. Since the thickness of the TEM specimen depends on the size, material and shape of the material (Muto et al., 2009; Sasaki et al., 2009), we prepared a cube specimen of about $10 \mu \mathrm{m} \times 10 \mu \mathrm{m} \times 10 \mu \mathrm{m}$ in a specific region using FIB and then prepared a TEM specimen preparation technique using ultramicrotome to obtain lamella. This technique (FIB-ultramicrotome technique) is expected to possess the advantages of both the FIB, which is favorable for sampling a specific area, and the ultramicrotome, which enables the preparation of thin specimens without Ga ion. In this study, two types of test specimens from the Si-based anode material of Li-ion battery was prepared by respectively adopting the FIB method only and the new FIB-ultramicrotome method. TEM analyses of two samples were conducted to compare the Ga ion damage of the test specimen.

\section{MATERIALS AND METHODS}

\section{Battery Manufacturing}

The manufacturing process of Si-based anode materials and battery, and the charge-discharge cycle testing method are the same as previous study (Chae et al., 2013). After 20 cycle testing, the battery was dismantled inside a glove box filled with inert Ar gas.

The lithium salts remaining on the surface was removed by using an organic solvent, dimethyl carbonate. The remaining materials were dried for approximately a day.

\section{TEM Specimen Preparation}

The prepared material was cracked and broken into shards through the volume expansion after undergoing the process as shown in Fig. 1. For TEM analysis, two types of specimen were prepared by using two kinds of preparation methods. The first method is a conventional TEM sampling method using FIB. Firstly, the FIB was used to select a specific area of interest from the shards of the charged materials. The selected area was thinned by FIB milling. In general, the thinner the thickness of the specimen, the better. But in this study, when the thickness of the specimen was too thin $(<70 \mathrm{~nm})$, the material properties were changed by ion-beam damage. The optimum thickness of our cross-sectional TEM specimen was 70 to $100 \mathrm{~nm}$. The second method is a new method using FIB and ultramicrotome, which is firstly proposed in this study. Initially, FIB was used to extract a cube sample of about $10 \mu \mathrm{m} \times 10 \mu \mathrm{m} \times 10 \mu \mathrm{m}$ from a specific area of interest. The extracted sample was attached to the top of the "B" post of lift-out grid for FIB in Fig. 2A. The grid was fixed to a support stick with glue, then placed in a plastic cap tube and filled with epoxy resin to make a block, as shown in Fig. 2A. The epoxy resin was prepared at a ratio of 4:1 resin to hardener. With a block being made, cautions should be taken to prevent bubble formation. The unintentionally formed microbubbles were removed through a vacuum defoamer followed by drying in a vacuum oven at $40^{\circ} \mathrm{C}$ for 24 hours. The block was then cut with a razor blade, in order to fit into a fixing holder. The block was smoothly trimmed into the size of about $50 \mu \mathrm{m} \times 50$ $\mu \mathrm{m}$ and located at the centered on the grid "B" post, to which the test piece was attached using a trimming blade (Fig. 2B). During the cutting in using the ultramicrotome, cutting rate was set as $0.8 \mathrm{~mm} / \mathrm{s}$ and as the thickness $70 \mathrm{~nm}$. A diamond blade $\left(3 \mathrm{~mm}, 35^{\circ}\right)$ was used to cut the sample trimmed in the prior step. Water, anhydrous ethanol and organic solvent were used as the solvents to float the severed specimen. In the case of anhydrous ethanol, the specimen was sank due to low specific gravity, and the organic solvent were solidified along the diamond blade and block in the boat. These caused difficulties in cutting and extracting the specimen. Therefore, water was used in this experiment. In order to minimize the reaction with $\mathrm{Li}$, the severed specimens were immediately

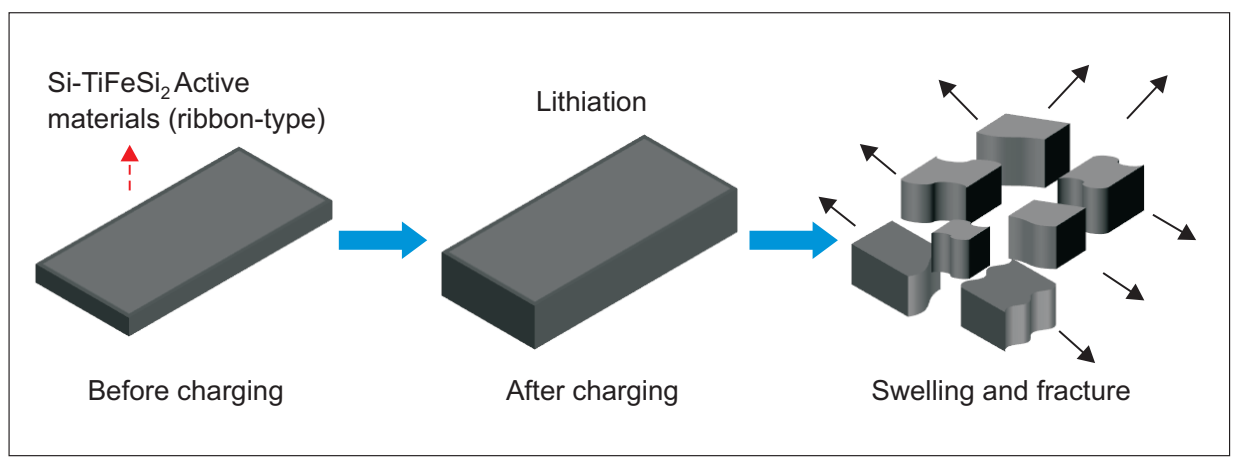

Fig. 1. Schematic illustration showing volume change process of $\mathrm{Si}-\mathrm{TiFeSi}_{2}$ active materials. 


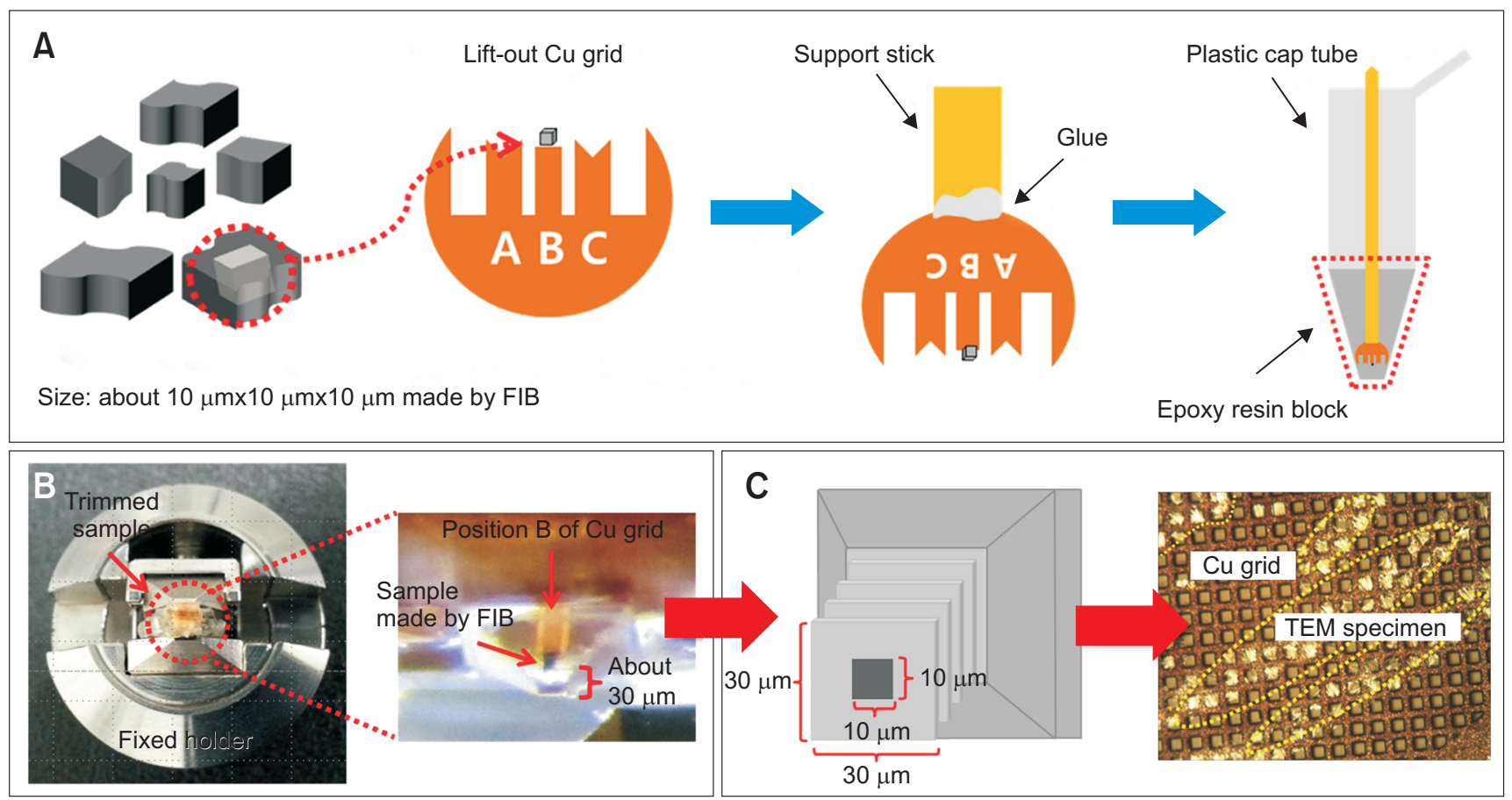

Fig. 2. Transmission electron microscope (TEM) sample preparation for charged Si-Ti-Fe anodes. (A) Sample molding process for ultramicrotomy. (B) A trimmed sample. (C) Fig. 2C continuous slices schematic diagram and optical microscope image and showing successive sections of sliced (as indicated by dotted ellipses) using ultramicrotome obtained from the trimmed sample. FIB, focused ion beam.

dried and used for TEM analysis.

\section{RESULTS AND DISCUSSION}

The material used in this study is a Si-based anode material comprising active material $\mathrm{Si}$ and $\mathrm{TiFeSi}_{2}$ matrix. The material was manufactured by melt-spinning method and composed of fine and coarse grain regions (Chae et al., 2013; Raghavan, 2009; Weitzer et al., 2008). In this study, the fine grain region was selected for the preparation of TEM specimens and was observed by TEM to confirm the Ga ion damage of the specimens.

TEM specimens made by the FIB-ultramicrotome technique were continuously obtained with the slice thickness of below $100 \mathrm{~nm}$. A slice thickness is affected by material, sample size, sample shape and trimmed surface area. In the conventional ultramicrotome technique, it is necessary to make many slices to secure large observation regions, because the region of interest cannot be exactly selected. However, since the FIBultramicrotome technique uses FIB to make the region of interest sampled, the trimming size can be reduced to achieve continuous slices that maintain thin slice thickness, as shown in Fig. 2C.

Fig. 3 shows TEM images and selected-area electron diffraction (SAED) patterns, obtained from the TEM specimens made by FIB and FIB-ultramicrotome technique, respectively
(Fig. 3A and B). Fig. 3C and D show the SAED patterns obtained from Fig. 3A and B. Both patterns showed the (312), (232) plane and (140) plane of the $\mathrm{TiFeSi}_{2}$ matrix. While SAED pattern of the specimen made by only FIB showed for $\mathrm{Si}$ to be amorphized by ion beam damage, SAED pattern of the specimen made by the FIB-ultramicrotome technique showed clearly $\mathrm{Si}$ (111) and $\mathrm{Li}_{21} \mathrm{Si}_{5}$ (620) plane. Typically, $\mathrm{Li}$-silicide is very weak to electron beam. However, the Lisilicide was stable during SAED analysis because it used a very low intensity of electron beam in widely expanded state. Therefore, it is considered that both $\mathrm{Si}$ and Li-silicide remain crystalline in the FIB-ultramicrotome specimen.

These results show that the FIB-ultramicrotome technique for TEM analysis can prepare the TEM specimen without artifacts such as the ion beam damage caused by FIB and plastic deformation and specimen tear by the ultramicrotome. To confirm the phase distributions of $\mathrm{Si}$ and $\mathrm{TiFeSi}_{2}$ matrix, an energy dispersive spectroscopy (EDS) mapping analysis was conducted. Fig. 4 shows the bright-field-TEM and EDS mapping images of specimens made by the only FIB and FIB-ultramicrotome technique, respectively. As a result, both samples showed two phases of $\mathrm{Si}$ and $\mathrm{TiFeSi}_{2}$ matrix, and it was confirmed that $\mathrm{TiFeSi}_{2}$ matrix was maintained even after charging.

In addition, in the specimen prepared by the only FIB technique, Ga elements were detected in the EDS analysis area, but 

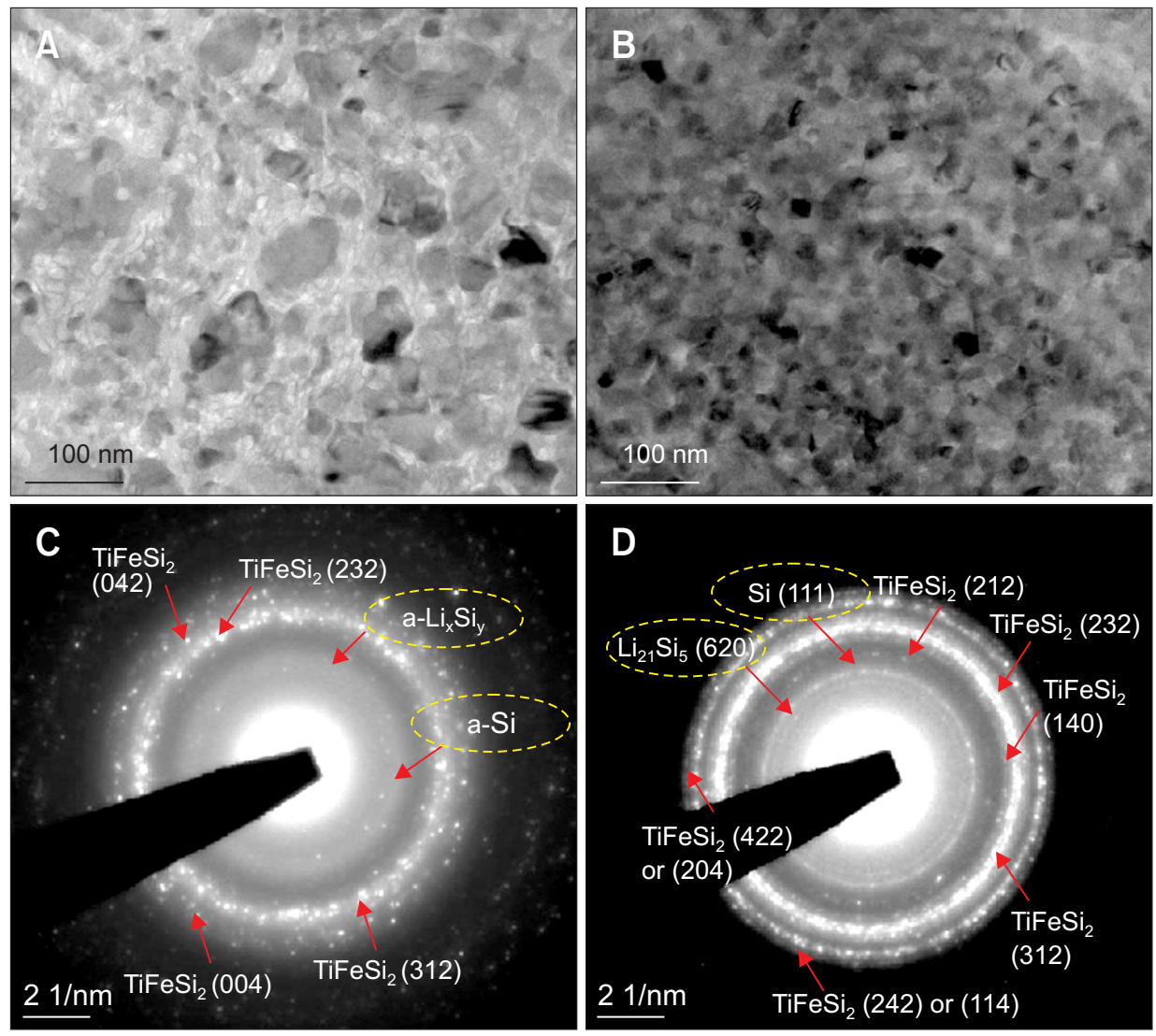

Fig. 3. Transmission electron microscope mages (A and B) and selected-area electron diffraction patterns ( $\mathrm{C}$ and $\mathrm{D})$ obtained from specimens prepared by focused ion beam (FIB) and FIB-ultramicrotome techniques, respectively.
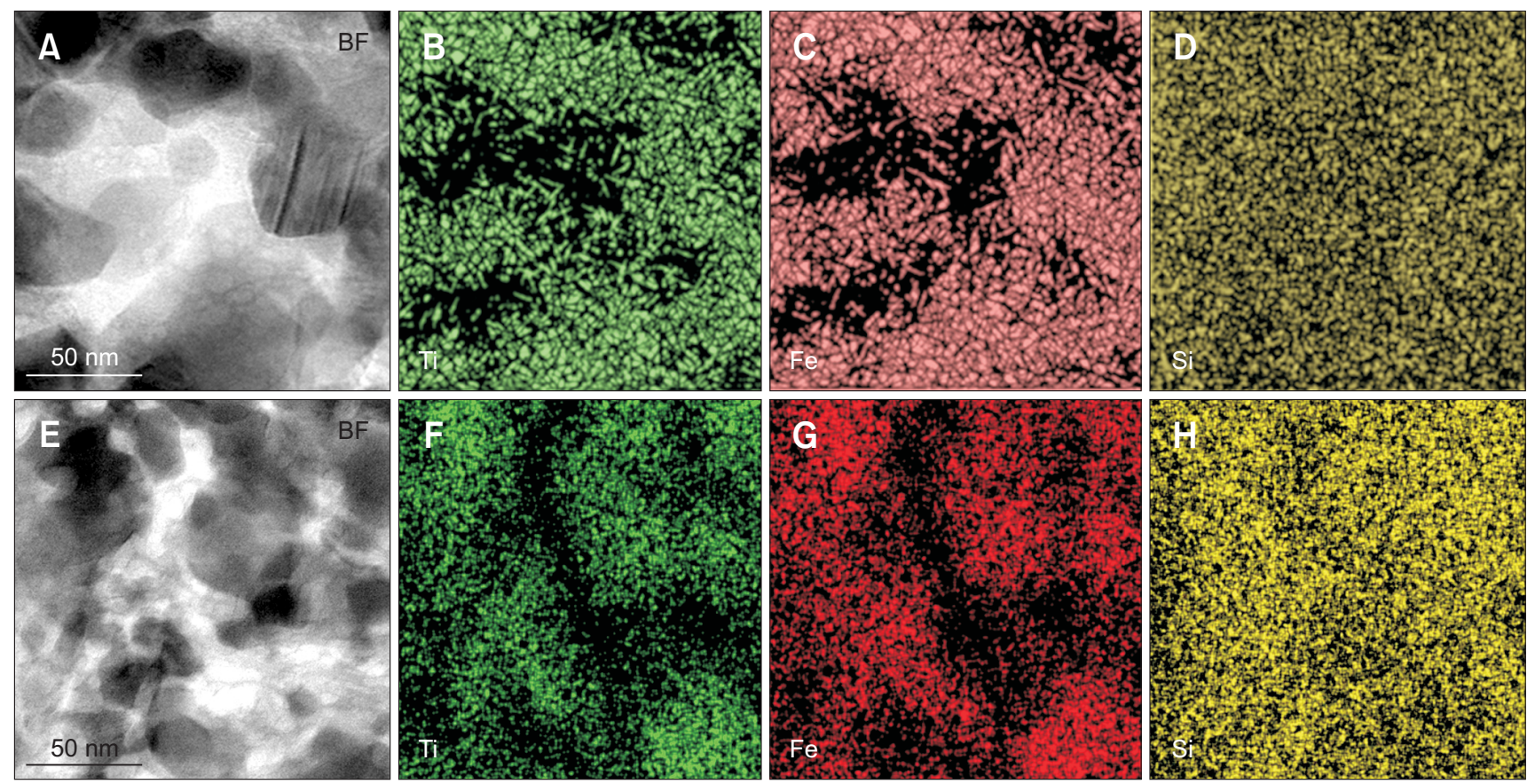

Fig. 4. Bright-field images (BF) and energy dispersive spectroscopy elemental mapping images of Ti, Fe, and Si for fine-grained regions. (A-D) Focused ion beam (FIB) and (E-H) FIB-ultramicrotome techniques, respectively. 
not detected in specimens prepared by FIB-ultramicrotome technique (not shown in this paper). It is thought that when the region of interest is extracted using the FIB, in FIBultramicrotome technique the surface of cube sample is damaged by the ion beam but does not affect the inside. It means that the FIB-ultramicrotome specimen shows no ion beam damage and then may can be suitable for EDS analysis. These results as shown in Fig. 3 and 4 prove that the TEM specimen prepared by the FIB-ultramicrotome technique is more preferable to analyze the microstructure of the Si-based anode material, compared with the TEM specimen made by the only FIB technique.

\section{CONCLUSIONS}

In this paper, FIB-ultramicrotome technique was proposed as a TEM specimen preparation method for a successful TEM analysis of the Si-based anode material. The specimen prepared by FIB-ultramicrotome technique was compared with that prepared by only FIB technique.

1. From the SAED patterns and the EDS mapping analysis, we estimated the extent of ion beam damage of the two specimens. The SAED pattern of the specimen made by only FIB showed amorphized $\mathrm{Si}$ and $\mathrm{Li}$-silicide by ion beam damage and the specimen made by new FIB-ultramicrotome technique showed clearly crystalline $\mathrm{Si}$ and Li-silicide. Also, in the specimen made by only FIB technique, Ga atoms were detected in EDS mapping area, but not in the specimen made by FIB-ultramicrotome technique.

2. By using the FIB-ultramicrotome technique, we can extract the specific area using FIB and cut continuous slices mechanically using an ultramicrotome without ion beam damages. In conclusion, this technique is expected to be very useful for preparing TEM specimen of not only Sibased anode materials, but also various materials which are weak to ion beams and in which specific areas need to be observed.

\section{CONFLICT OF INTEREST}

No potential conflict of interest relevant to this article was reported.

\section{ACKNOWLEDGMENTS}

This research was supported by the MOTIE (Ministry of Trade, Industry \& Energy) (10080654) and KSRC (Korea Semiconductor Research Consortium) support program for the development of the future semiconductor device.

\section{REFERENCES}

Chae J E, Yang J M, Park K J, Yoo J H, Park Y C, Sung M S, Yu H J, and Kim S S (2013) Microstructural analysis of Si-Ti-Fe alloy anode materials for Li-ion secondary batteries. Korean J. Met. Mater. 51, 429-436.

Chan C K, Peng H, Liu G, Mcllwrath K, Zhang X F, Huggins R A, and Cui $Y(2008)$ High-performance lithium battery anodes using silicon nanowires. Nat. Nanotechnol. 3, 31-35.

Danet J, Brousse T, Rasim K, Guyomard D, and Moreau P (2010) Valence electron energy-loss spectroscopy of silicon negative electrodes for lithium batteries. Phys. Chem. Chem. Phys. 12, 220-226.

Jung H, Kim Y U, Sung M S, Hwa Y, Jeong G, Kim G B, and Sohn H J (2011) $\mathrm{Si}$ anode embedded in super-elastic nitinol (Ni-Ti) shape memory alloy matrix for Li rechargeable batteries. J. Mater. Chem. 21, 1121311216.

Lee H Y and Lee S M (2002) Graphite-FeSi alloy composites as anode materials for rechargeable lithium batteries. J. Power Sources 112, 649-654.

Mao O, Turner R L, Courtney I A, Fredericksen B D, Buckett M I, Krause L J, and Dahn J R (1999) Active/inactive nanocomposites as anodes for Li-ion batteries. Electrochem. Solid-State Lett. 2, 3-5.

Muto S, Sasano Y, Tatsumi K, Sasaki T, Horibuchi K, Takeuchi Y, and Ukyo
Y (2009) Capacity-fading mechanisms of $\mathrm{LiNiO}_{2}$-based lithium-ion batteries(II). J. Electrochem. Soc. 156, 371-377.

Obrovac M N and Christensen L (2004) Structural changes in silicon anodes during lithium insertion/extraction. Electrochem. Solid-State Lett. 7, A93-A96.

Park C M, Kim J H, Kim H, and Sohn H J (2010) Li-alloy based anode materials for Li secondary batteries. Chem. Soc. Rev. 39, 31153141.

Raghavan V (2009) Fe-Si-Ti (iron-silicon-titanium). J. Phase Equilibria Diffus. 30, 393-396.

Sasaki T, Nonaka T, Oka H, Okuda C, Itou Y, Kondo Y, Takeuchi Y, Ukyo Y, Tatsumi K, and Muto S (2009) Capacity-fading mechanisms of $\mathrm{LiNiO}_{2}$-based lithium-ion batteries(I). J. Electrochem. Soc. 156, 289293.

Weitzer F, Schuster J C, Naka M, Stein F, and Palm M (2008) On the reaction scheme and liquidus surface in the ternary system Fe-SiTi. Intermetallics 16, 273-282.

Winter M, Besenhard J O, Spahr M E, and Novák P (1998) Insertion electrode materials for rechargeable lithium batteries. Adv. Mater. 10, 725-763. 